Increasing evidence suggests that the steroid hormone testosterone (T) enhances libido and decreases depression. Even a single administration of $T$ (0.5 mg sublingually) in healthy young women is sufficient to enhance physiological sexual responsiveness. Such physiological evidence is not yet available for the link between $T$ and depression. Recent research has revealed that lowered functional connectivity in a specific corticocortical pathway may be a sensitive physiological index for depression. This pathway, comprised of the left prefrontal and right parietal cortex, has been named a cortical depression circuit. In the present study, a single dose of $T$ was administered to healthy young women to investigate the effects on the functional connectivity in this corticocortical depression circuit. It was hypothesized that administration of $T$ would lead to an increase of functional connectivity. In a double-blind placebo-controlled, crossover design, fourteen healthy females received (sublingually) a single dose of $0.5 \mathrm{mg} \mathrm{T}$ or placebo in a randomly assigned fashion. Three hours after drug administration the functional coupling between the left prefrontal and right parietal cortex was established by measuring the interhemispheric electroencephalogram (EEG) coherence for the different

\section{Administration of Testosterone Increases Functional Connectivity in a Cortico-Cortical Depression Circuit}

\author{
Dennis J.L.G. Schutter, Ph.D. \\ Jiska S. Peper, M.S. \\ Hans P.F. Koppeschaar, M.D., Ph.D. \\ René S. Kahn, M.D., Ph.D. \\ Jack van Honk, Ph.D.
}

frequency bands. Compared to placebo, T administration significantly increased the functional connectivity in the $\sigma(1-3 \mathrm{~Hz})$ frequency range between the left prefrontal and right parietal cortex. Reductions in interhemispheric coherence in the $\sigma$ frequency range have been observed in clinically depressed patients. Thus the present findings may provide a first insight into the neurobiological mechanism by which $T$ decreases depression. The fact that only a single dose of $T$ was able to induce the effect in healthy female subjects suggests that the mechanism is highly sensitive. A feasible application of $T$ treatment in the struggle against depression is discussed.

(The Journal of Neuropsychiatry and Clinical Neurosciences 2005; 17:372-377)

\footnotetext{
Received July 14, 2003; revised April 5, 2004; accepted April 6, 2004. From the Helmholtz Research Institute, Utrecht University, Utrecht, the Netherlands; the Department of Endocrinology, University Medical Centre, Utrecht, the Netherlands; and the Department of Psychiatry, University Medical Centre, Utrecht, the Netherlands. Address correspondence to Dr. Schutter, Helmholtz Research Institute, Affective Neuroscience Section, Utrecht University, Heidelberglaan 2, 3584 CS Utrecht, the Netherlands; D.Schutter@fss.uu.nl (E-mail). Copyright (C) 2005 American Psychiatric Publishing, Inc.
} 
$S_{\mathrm{h}}^{\mathrm{e}}$ veral studies have demonstrated that the steroid hormone testosterone $(\mathrm{T})$ improves well-being especially in women ${ }^{1}$ and decreases depression. ${ }^{2}$ However, the research focus on well-being has mainly been on sexual desire and, in particular, a decline in sexuality after a drop in androgenic functions has been demonstrated. Relatively low levels of $\mathrm{T}$ have been associated with reductions in coital frequency and diminished sexual desire. ${ }^{3,4} \mathrm{~T}$ substitution alone in androgenic deficient women seems, however, not sufficient to completely restore sexual functioning. A discrepancy has been observed in woman suffering from hypothalamic amenorrhea, whereby sexual physiological arousal increased after $\mathrm{T}$ treatment, but the subjectively measured excitement remained unchanged. ${ }^{5}$ It was suggested that $\mathrm{T}$ treatment should be accompanied by appropriate psychotherapeutical interventions. Two fundamental studies have provided insights into the mechanisms by which $\mathrm{T}$ enhances sexual functioning. A transient effect on both subjective and physiological sexual arousal with a time course of 3-4 hours was shown after a single administration of $\mathrm{T}$ in healthy young women. ${ }^{6}$ Interestingly, a further study revealed that the effect of $\mathrm{T}$ on the subjective component in the latter study was a priming confound of the repeated measurements in search for this time course. ${ }^{7}$ This finding again reveals the earlier observed discrepant effects of $\mathrm{T}$ administration on subjective and physiological sexual functioning. ${ }^{5}$ The above noted fundamental research has not been done with respect to the proposed link between $\mathrm{T}$ and depression. ${ }^{1}$

Evidence suggests that the left prefrontal (PFC) and the right parietal cortex are dysfunctional in depression..$^{8-10}$ A sensitive electrophysiological marker for depression was, however, missing until recently. Interestingly, endocrinological studies in healthy human volunteers have demonstrated that a biochemical marker for depression, heightened baseline levels of the stress-hormone cortisol. ${ }^{11}$ are related to lowered functional connectivity between the left PFC and right parietal cortex. ${ }^{12}$ A further study in healthy volunteers showed that cortisol was indeed associated with depressive mood, ${ }^{13}$ as often demonstrated in clinically depressed patients. ${ }^{11}$ Together, these findings suggest a sensitive relationship between the functional connectivity (cross talk) in the left prefrontal-right parietal cortico-cortical circuit and depression. In such a way that lowered functional connectivity in the latter brain circuit might predispose to depression. Findings from a brain stimulation technique, repetitive transcranial magnetic stimulation
(rTMS), seem to provide further evidence for the existence of such a left prefrontal-right parietal corticocortical depression circuit. Note that the effects of rTMS depend on stimulation parameters, whereas the fast frequency rTMS $(>5 \mathrm{~Hz})$ produces neural excitation, the slow frequencies cause neural inhibition of the targeted regions. ${ }^{14,15}$ The antidepressant effects of fast rTMS over the left PFC are concordantly argued to be due to normalization of hypometabolism in the left PFC. ${ }^{8}$ This may, however, not be the main mechanism responsible. Importantly, a study of Jing and Takigawa ${ }^{16}$ demonstrated an increase in functional connectivity in the above noted left prefrontal-right parietal cortico-cortical depression circuit after high frequency rTMS over the left PFC. Increases in functional connectivity between different cortical regions have also been demonstrated after slow rTMS. ${ }^{17}$ Crucially, Rosenberg et al. ${ }^{18}$ showed comparable antidepressant efficacy after both slow and fast rTMS over the left PFC. The latter findings are paradoxical in terms of the traditional normalization of left PFC hypometabolism claim, since slow rTMS should in fact further decrease left PFC metabolism. ${ }^{14,15}$ However, findings in the Rosenberg et al. ${ }^{18}$ study can be interpreted in terms of the strengthening of the functional connectivity in the left prefrontal-right parietal corticocortical depression circuit by both slow and fast rTMS. ${ }^{19}$ Finally, working from a heuristic model constituting this depression circuit, and assuming that rTMS would also be capable of strengthening functional connectivity when stimulating the right parietal cortex, a recent placebo controlled study by Van Honk and Schutter ${ }^{20}$ demonstrated reductions in phenomenological, attentional and physiological indices of depression in healthy volunteers after slow rTMS over the right parietal cortex.

In sum, there are indications that $\mathrm{T}$ has antidepressant efficacy, ${ }^{1}$ but physiological evidence is lacking. In search for such evidence we investigated whether a single administration of $\mathrm{T}$ in healthy young women would be capable of strengthening the functional connectivity in the left prefrontal-right parietal cortico-cortical depression circuit.

\section{METHOD}

\section{Participants}

Volunteers were recruited among students at Utrecht University, Utrecht, The Netherlands. Fourteen non- 
TESTOSTERONE AND A CORTICO-CORTICAL DEPRESSION CIRCUIT

smoking, healthy, right-handed women participated in the study $($ mean $=21.6$ years, $S D=1,9)$. We studied healthy, young females: standard clinical interview revealed that they had no (family) history of psychiatric/ neurological or endocrine diseases, making a subclinical endocrine disorder or a pre-symptomatic thyroid disorder highly unlikely. They all used low dose sub-30 oral contraceptives, containing 20 microgram ethinylestradiol and 150 microgram desogestrel (as progestin) once a day. All measurements both during placebo and $\mathrm{T}$ were done within the first week after starting the oral contraceptive. During the use of an oral contraceptive there is no menstrual phase (LH and FSH are suppressed to prevent ovulation), but depending on the formula of the oral contraceptive one can provoke once a month a withdrawal bleeding during which no active hormone is given. Only women participated because the time course and the dosage of T necessary to establish physiological and psychological effects after a single administration have yet not been established in men. ${ }^{6,13}$ All volunteers were unaware of the aim of the study. Informed consent was obtained and participants received payment for taking part in the study. The protocol was approved by the Medical Ethics Committee of the University Medical Centre in accordance with the Declaration of Helsinki.

\section{Procedure}

Participants were tested twice in a double blind, placebocontrolled crossover design. Both testing days were separated by at least 48 hours. Prior to experimentation, the procedure was explained in full detail and participants were instructed to refrain from using medication and psychoactive drugs, such as coffee and alcohol on both testing days. Eating was not allowed 1 hour prior to each session. In the morning, between 8.30 and 10.30 a.m., upon arrival at the Psychological Laboratory of the Utrecht University, volunteers sublingually received 0.5 mg of either T with cyclodextrines as carrier or placebo. Recent experimental studies in our laboratory have established the time course of $0.5 \mathrm{mg}$ sublingual T administration on blood levels and physiological responsivity. These studies showed without exception at least a tenfold increase in the levels of total $\mathrm{T}$ in plasma (with no changes in binding globulin) fifteen minutes after intake, with a return to baseline within ninety minutes. ${ }^{6}$ Furthermore, it was repeatedly observed that this single dose of T significantly elevated different indices of physiological responsivity approximately 3 to 4 hours after intake. ${ }^{6,7,13}$ This time course effect of $\mathrm{T}$ administration on subjective and physiological arousal was presently taken into account. Three hours after $\mathrm{T}$ administration, volunteers returned to the laboratory where the mood states, i.e. depression, anger and anxiety were assessed. After completion of the mood inventory, EEG recording preparations were made, which took on average about thirty minutes. Participants were seated in a comfortable chair in a dimly lit, quiet room, while the experimenter was in an adjacent control room. To obtain baseline measurements of background EEG, subjects were instructed to stay awake and to sit as motionless as possible. Oneminute intervals of background EEG with eyes-open $(\mathrm{O})$ and eyes-closed (C) for a total of four minutes (Sequence: O-C-O-C) were recorded $3 \frac{1}{2} 2$ hours after $\mathrm{T}$ administration.

\section{Dependent Measures}

Baseline EEGs were recorded from twelve scalp positions (Fz, Fp1, Fp2, F3, F4, Cz, C3, C4, Pz, P3, P4, Oz) according to the International 10/20 System of EEG electrode positions, using an electro-cap with Ag/AgClelectrodes (Neurosoft, Inc.). The reference electrode was placed on the right mastoid. Electro-oculogram (EOG) was recorded by placing $\mathrm{Ag} / \mathrm{AgCl}$ electrodes to the supra- and suborbit of the right eye and on the external canthi of each eye, in order to correct for vertical and horizontal eye movements. ECI EEG gel was used as conducting medium for both EEG and EOG electrodes and all impedances were lower than 5,000 Ohms. An acquisition amplifier (Ampligraph) was used to filter incoming signals (low pass cut-off frequency was $70 \mathrm{~Hz}$ with a time constant of $3 \mathrm{~s}$ ). Amplification was set at 20,000 for both the EEG and EOG leads, and the sample rate was set at $250 \mathrm{~Hz}$. For the actual EEG registration, recording and data processing, Neuroscan Software (El Paso, Texas) was used. Raw EEG signals were digitally filtered offline with a 1-30 Hz bandpass filter setting. EEG signal containing eye and/ or muscle movements, or other forms of artifacts, greater than $-50 \mu \mathrm{V}$ and +50 $\mu \mathrm{V}$ were rejected for further analysis. After artifacts were discarded, data were corrected for horizontal and vertical eye movements using linear regression analysis. For the quantitative analysis of interhemispheric coherence, one electrode pair of interest, i.e. the functional connectivity between the left prefrontal and right parietal areas was selected on the basis of our a priori hypothesis, which states that $\mathrm{T}$ should increase cross talk between these two cortical regions. The four frequency 
bands were extracted using a fast Fourier transform algorithm (Hamming window: length 10\%). Cross-spectra for the different frequency bands were calculated and normalized by the autospectra to compute coherence, according to the following equation:

$$
\left|R_{x y}(e)\right|^{2}=\frac{\left|G_{x y}(e)\right|^{2}}{G_{x x}(e)+G_{y y}(e)}
$$

In the equation, $G_{x x}(e), G_{y y}(e)$ and $G_{x y}(e)$ are values of the auto- and cross-spectra at a given frequency band $e$ respectively. Coherence indexes the linear synchronization between electrode pairs and ranges from absence (0) to perfect (1) association. The fronto-parietal coherence was determined for the $\sigma(1-3 \mathrm{~Hz}), \theta(4-7 \mathrm{~Hz}), \alpha$ (8-13 Hz) and $\beta$ (14-30 Hz) frequency bands.

Self-reports on state depression, anger and anxiety were quantified using a visual analogue scale computerized version of the POMS (Profile of Mood States) questionnaire. $^{21}$

\section{Statistical Analyses}

Separate multivariate analysis of variance (MANOVAs) for repeated measurements with Greenhouse-Geisser corrected $p$-values were carried out for the $\sigma, \theta, \alpha, \beta$ frequency bands as within-subject variables and with Order of drug intake as between-subjects factor, respectively. In order to detect possible changes in mood states as a result of drug, separate MANOVAs for repeated measurements with Greenhouse-Geisser corrected p-values were performed with depression, anger and anxiety as withinparticipants variables and Order of drug intake as between-participants factor. The $\alpha$ level of significance (two-tailed) was $\mathrm{p}<0.05$ throughout.

\section{RESULTS}

Electrophysiological Data

Multivariate testing revealed a statistical significant increase in interhemispheric coherence in the $\sigma$ frequency range after $\mathrm{T}$ compared to placebo administration $[\mathrm{F}(1,12)=7,4: \mathrm{p}<0.02]$. There was no Drug $\times$ Order effect ( $p>0.8)$, indicating no carry-over effects of administration. No significant changes after $\mathrm{T}$ administration were found for the $\theta, \alpha, \beta$ frequency bands ( $p>$ $0.5)$. Figure 1 displays the significant increase in left prefrontal (F3) and right parietal (P4) $\sigma$ coherence and the coherence values for the $\theta, \alpha$, and $\beta$ frequency bands after $0.5 \mathrm{mg}$ of $\mathrm{T}$ and placebo.

\section{Mood States}

Separate MANOVAs were used to investigate possible effects of $0.5 \mathrm{mg} \mathrm{T}$ on self-reported mood states of depression, anger and anxiety. Statistical analyses did not show any significant changes in mood ( $p>0.5)$. In Table 1 the means and standard errors of the mean (SEM) are shown for the dependent variables in the $\mathrm{T}$ and placebo condition.

\section{DISCUSSION}

This study investigated whether a single administration of $\mathrm{T}$ would increase the functional connectivity between the left PFC and right parietal cortex. In line with our hypothesis, $\mathrm{T}$ as compared to placebo induced a significant increase in the functional connectivity in this left prefrontal-right parietal cortico-cortical depression circuit. ${ }^{19}$ Although EEG coherence between different cortical regions is largely established by cortico-cortical and thalamo-cortical interactions, ${ }^{22}$ subcortical brain areas contribute to both inter- and intrahemispheric functional communication as well. ${ }^{23}$ Especially the lower bandwidths such as the $\sigma$ frequency in the EEG coherence spectrum have been associated with limbic contributions to cortico-cortical

FIGURE 1. Means ( \pm SEM) For the Left Frontal-Right Parietal (F3-P4) Coherence in the $\sigma, \theta, \alpha$, and $\beta$ Frequency Bands in the $\mathrm{T}$ and Placebo Condition

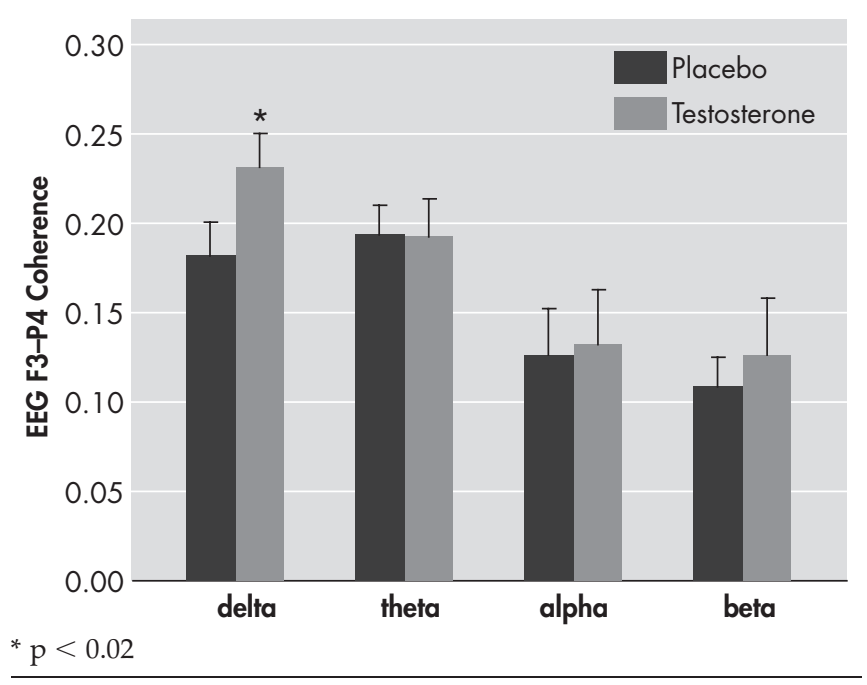


coupling. ${ }^{24}$ Interestingly, the hormone $\mathrm{T}$ is argued to establish its effects on emotional processing by binding to specific steroid responsive networks in the limbic system. ${ }^{25}$ Presently, the T-induced changes in these networks may have led to a cascade of biochemical events resulting in the strengthening of the functional connectivity in the $\sigma$ frequency band of the left prefrontalright parietal depression circuit. ${ }^{26}$ In crucial defense of this notion, a study by Roemer et al. ${ }^{27}$ demonstrated lower interhemispheric coherence in the $\sigma$ frequency range in depressive patients compared to healthy controls. The authors suggested subcortical abnormalities to be responsible for the lowered coherence.

The absence of effects on self-reported depressive mood in the present study concurs with earlier research whereby single doses of $0.5 \mathrm{mg} \mathrm{T}$ administration in healthy human volunteers induced a similar discrepancy between physiological and self-reported variables. ${ }^{7,13}$ Moreover, as already noted in the Introduction, the same discrepancies have also been observed in a clinical $\mathrm{T}$ treatment study. ${ }^{5}$ Apparently, $\mathrm{T}$ induces changes in physiological affective processing which cannot easily be captured by self-report. ${ }^{28}$

Importantly, it was recently demonstrated that methodiogically amygdala responses to masked fearful faces normalized after antidepressant treatment. ${ }^{29}$ Strikingly, in a placebo controlled cross-over $\mathrm{T}$ administration study, similar to the present study, we observed concurring reductions in affective responses to masked fearful faces. ${ }^{30}$ This provides further evidence for T's antidepressant properties and points at the likelihood of the involvement of limbic structures in these effects of $\mathrm{T}$. The search for other regions of interest was not the aim of the present study, but future $\mathrm{T}$ administration research might look at other cortical regions involved.

Finally, of interest from a clinical perspective, it has been demonstrated that higher pretreatment interhemispheric coherence in the $\sigma$ frequency range in clinically depressed subjects results in a more positive therapeutical outcome of electroconvulsive therapy (ECT). Hence, the present findings, when taken together with earlier reports of $\mathrm{T}$ administration ${ }^{5-7}$ suggest that $\mathrm{T}$ may lay a neurochemical basis for successful therapy. The strengthening of the functional connectivity in the $\sigma$ frequency range by $\mathrm{T}$ treatment in depressed individuals might, for instance, increase the success of behavioral and psychotherapeutical interventions. ${ }^{5}$ Moreover, in future research it might be worthwhile to investigate first degree family history with respect to the assumed relationship between $\sigma$ coherence and depressive mood disorders. At this moment we are preparing clinical trials to test the hypothesis whether $\mathrm{T}$ treatment in combination with psychotherapy has enhanced antidepressant efficacy.

This study was sponsored by an Innovational Research Grant (\# 016-005-060) from the Netherlands Organization for Scientific Research (NWO).

TABLE 1. Means and Standard Errors of the Mean (SEM) for Dependent Variables in the T and Placebo Condition

\begin{tabular}{lcccccrr}
\hline & $\boldsymbol{\sigma}$ & $\boldsymbol{\theta}$ & $\boldsymbol{\alpha}$ & $\boldsymbol{\beta}$ & Depression & Anger & Anxiety \\
\hline T & $0.23 \pm 0.02$ & $0.19 \pm 0.02$ & $0.13 \pm 0.03$ & $0.13 \pm 0.03$ & $-4.00 \pm 2.25$ & $-1.93 \pm 1.68$ & $-0.21 \pm 1.43$ \\
Placebo & $0.18 \pm 0.02$ & $0.19 \pm 0.02$ & $0.13 \pm 0.03$ & $0.11 \pm 0.02$ & $-7.35 \pm 3.27$ & $-5.93 \pm 3.67$ & $-1.07 \pm 1.71$ \\
\hline
\end{tabular}

\section{References}

1. Davis SR, Tran J: Testosterone influences libido and well being in women. Trends Endocrinol Metab 2000; 12:33-37

2. Rabkin JG, Wagner GJ, Rabkin R: Testosterone therapy for human immunodefiency virus-positive men with and without hypogonadism. J Clin Psychopharmacology 1999; 19:19-27

3. Bachmann GA, Leiblum SR: Sexuality in sexagenarian women. Maturitas 1991; 13:43-50

4. McCoy NL, Davidson JM: A longitudinal study of the effects of menopause on sexuality. Maturitas 1985; 7:203-210

5. Tuiten A, Laan E, Panhuysen G, et al: Discrepancies between genital responses and subjective sexual function during testosterone substitution in women with hypothalamic amenorrhea. Psychosom Med 1996; 58:234-241
6. Tuiten A, Van Honk J, Koppeschaar HPF, et al: Time course of effects of testosterone administration on sexual arousal in women. Arch Gen Psychiatry 2000; 57:149-153

7. Tuiten A, Van Honk J, Verbaten R, et al: Can sublingual testosterone increase subjective and physiological measures of laboratory-induced sexual arousal? Arch Gen Psychiatry 2002; 59: 465-466

8. George MS, Wassermann EM, Kimbrell TA, et al: Mood improvement following daily left prefrontal repetitive transcranial magnetic stimulation in patients with depression: a placebocontrolled crossover trial. Am J Psychiatry 1997; 154:1752-1756

9. Davidson RJ: Affect, cognition, and hemispheric specialization, in Emotions, Cognition, and Behavior, edited by Izard CE, Ka- 
gan J, Zajonc RB. Cambridge, Cambridge University Press, 1984, pp 320-365

10. Flor-Henry P: On certain aspects of the localization of the cerebral systems regulating and determining emotion. Biol Psychiatry $1979 ; 14: 677-698$

11. Holsboer F: The corticosteroid receptor hypothesis of depression. Neuropsychopharmacology 2000; 23:477-501

12. Schutter DJLG, Van Honk J, Koppeschaar HPF, et al: Cortisol and reduced interhemispheric coupling between the left prefrontal and right parietal cortex. J Neuropsychiatry Clin Neurosci 2002; 14:89-90

13. Van Honk J, Tuiten A, Hermans E, et al: A single administration of testosterone induces cardiac accelerative responses to angry faces in healthy young women. Behav Neurosci 2001; 115:238-242

14. George MS, Lisanby SH, Sackeim HA: Transcranial magnetic stimulation: applications in neuropsychiatry. Arch Gen Psychiatry 1999; 56:300-311

15. Wassermann EM, Lisanby SH: Therapeutic application of repetitive transcranial magnetic stimulation: a review. Clin Neurophysiol 2001; 112:1367-1377

16. Jing H, Takigawa M: Observation of EEG coherence after repetitive transcranial magnetic stimulation. Clin Neurophysiol 2000; 111:1620-1631

17. Strens LH, Oliviero A, Bloem BR, et al: The effects of subthreshold $1 \mathrm{~Hz}$ repetitive TMS on cortico-cortical and interhemispheric coherence. Clin Neurophysiol 2002; 113:1279-1285

18. Rosenberg PB, Mehndiratta RB, Mehndiratta YP, et al: Repetitive transcranial magnetic stimulation treatment of comorbid posttraumatic stress disorder and major depression. J Neuropsychiatry Clin Neurosci 2002; 14:270-276

19. Schutter DJLG, D'Alfonso AAL, Van Honk J: Counterintuitive antidepressant properties of slow rTMS over the left frontal cortex: a possible mechanism. J Neuropsychiatry Clin Neurosci 2003; 15:243-244

20. Van Honk J, Schutter DJLG, Putman P, et al: Reductions in phenomenological, physiological and attentional indices of depres- sion after $2 \mathrm{~Hz}$ rTMS over the right parietal cortex. Psychiatry Res 2003; 120:95-101

21. Shacham S: A shortened version of the Profile of Mood States. J Pers Assess 1987; 47:305-306

22. Nunez PL, Srinivasan R, Westdorp AF, et al: EEG coherency I: statistics, reference electrode, volume conduction, Laplacians, cortical imaging, and interpretation at multiple scales. Electroencephalogr Clin Neurophysiol 1997; 103:499-515

23. Davey MP, Victor JD, Schiff ND: Power spectra and coherence in the EEG of a vegetative patient with severe asymmetric brain damage. Clin Neurophysiol 2000; 111:1949-1954

24. Locatelli T, Cursi M, Liberati D, et al: EEG coherence in Alzheimer's disease. Electroencephalogr Clin Neurophysiol 1998; 106: 229-237

25. Wood RI: Functions of the steroid-responsive neural network in the control of male hamster sexual behavior. Trends Endocrinol Metab 1996; 7:338-344

26. Cottingham S, Phaff D: Interconnectedness of steroid hormonebinding neurons: Existence and implications. Curr Top Neuroendocrinol 1986; 7:223-249

27. Roemer RA, Shagass C, Dubin W, et al: Relationship between pretreatment electroencephalographic measures and subsequent response to electroconvulsive therapy: A preliminary study. Neuropsychobiology 1990; 24:121-124

28. Gray A, Jackson DN, McKinlay JB: The relation between dominance, anger, and hormones in normally aging men: results from the Massachusetts Male Aging Study. Psychosom Med 1991; 53:375-385

29. Sheline YI, Barch DM, Donnelly JM, et al. 2001. Increased amygdala response to masked emotional faces in depressed subjects resolves with antidepressant treatment: an fMRI study. Biol Psychiatry 2001; 50:651-681

30. Van Honk J, Peper JS, Schutter DJLG: Testosterone reduces unconscious fear but not consciously experienced anxiety: Implications for the disorders of fear and anxiety. Biol Psychiatry 2005; $58: 218-225$ 